\title{
Decreased trabecular bone deterioration of proximal tibiae and lumbar vertebrae in postmenopausal women with osteoarthritis
}

\author{
ZHENHUA YIN, WEI WANG, JIAN HAN, KE CHEN and JIANJUN MA \\ Department of Orthopedics, The First People's Hospital, Xianyang, Shaanxi 712000, P.R. China
}

Received November 27, 2015; Accepted November 10, 2016

DOI: $10.3892 /$ etm.2017.4439

\begin{abstract}
Osteoporosis and osteoarthritis (OA) are two common age-related skeletal disorders, which are associated with substantial morbidity and disability, particularly among elderly women. The present study was performed to investigate the trabecular bone deterioration of proximal tibiae and lumbar vertebrae in postmenopausal women with OA. The results demonstrated that the histomorphometric section of trabecular bone below the growth plate was markedly different between the healthy control and OA group. However, the loss of trabecular bone underneath the growth plate in the healthy control group was significantly worse than that of the OA group. Hematoxylin and eosin staining demonstrated the increased disconnection and separation of the trabecular bone network as well as the reduction of trabecular bone mass of primary and secondary spongiosa throughout the proximal metaphysis of tibia in the healthy control compared to the OA group; similar results were found when the same experiment was repeated on the lumbar vertebrae of healthy control subjects and OA patients from postmenopausal women. The biological properties of trabecular bone in the proximal tibia and lumbar vertebrae were measured in postmenopausal women with OA. Spearman's rank correlation analysis revealed that the bone volume fraction was both positively correlated with radiographic severity and Western Ontario McMaster University Osteoarthritis Index scores in in the proximal tibia and lumbar vertebrae from postmenopausal women with OA. In conclusion, the structural properties of the proximal tibia and lumbar vertebrae supported that an inverse correlation existed between postmenopausal women with OA and healthy controls. Moreover, there is an important protective mechanism of OA on trabecular microstructure in proximal tibiae and lumbar vertebrae from postmenopausal women.
\end{abstract}

Correspondence to: Dr Jianjun Ma, Department of Orthopedics, The First People's Hospital, 10 Biyuan Road, Xianyang, Shaanxi 712000, P.R. China

E-mail: fh_majj@163.com

Key words: osteoarthritis, postmenopausal osteoporosis, bone morphometry, trabecular bone

\section{Introduction}

Osteoporosis (OP) and osteoarthritis (OA) are two common age-related skeletal disorders, which are associated with substantial morbidity and disability, particularly among elderly women (1). Low bone mineral density (BMD) is reported in patients with early ankylosing spondylitis and rheumatoid arthritis $(2,3)$. However, reports of increased risk of bone deterioration in patients with OA remain controversial $(4,5)$. The correlation between $\mathrm{OA}$ and bone fragility has been subject to a considerable number of studies $(6,7)$. Epidemiological surveys suggest that $\mathrm{OA}$ and $\mathrm{OP}$ are rarely present together in the same patient $(1,8)$. In addition, cross-sectional studies have indicated that $\mathrm{OA}$ is associated with an increase in $\operatorname{BMD}(1,9)$, leading to a common assumption that an increased BMD is protective against OA progression. This has been demonstrated in postmenopausal women with OA undergoing total hip arthroplasty (THA), also revealing that the trabecular microarchitecture of the femoral neck in postmenopausal osteoarthritic subjects is enhanced compared with age-matched healthy controls $(10,11)$. Contrastingly, the presence of OP within the femoral head in OA patients has been confirmed, and OA does not appear to protect a patient from generalized primary OP $(12,13)$.

Postmenopausal osteoporosis (PMO) is characterized by excessive bone resorption, which also occurs at the early stages in the development of OA (14). However, increased bone formation, subchondral sclerosis and osteophyte formation take place in both the early and late stages of OA development (15). A previous study demonstrated significant differences in the trabecular bone, collagen fibers, lacunae and osteoblasts between PMO and OA in the femoral head (16). These observations support the hypothesis that there is an inverse correlation between OP and OA $(11,14,17)$. Moreover, an inverse correlation between vertebral fractures and spine OA in postmenopausal women with OP is reported (18). To the best of our knowledge, there has been no study of patients with knee OA who may be at risk of OP due to trabecular bone deterioration of the proximal tibiae and lumbar vertebrae. Therefore, the aim of the present study was to investigate trabecular bone deterioration of proximal tibiae and lumbar vertebrae in postmenopausal women with OA by two-dimensional analysis, and to provide further proof to support the idea that there is an inverse correlation between knee OA and OP in postmenopausal women. 


\section{Materials and methods}

Patients and specimens. The present study included a total of 20 subjects (10 used as healthy controls and 10 patients with OA). Subjects were recruited from September, 2012 to December, 2014 from The First People's Hospital in Xianyang, China, and osteoarthritic bone specimens were taken from postmenopausal women suffering from primary hip arthrosis undergoing THA. The diagnosis of primary OA referred to the criteria of the American College of Rheumatology (19). In both groups, patients with illnesses that may affect bone metabolism, including hyperparathyroidism, hyperthyroidism, osteomalacia, renal dysfunction and diabetes mellitus or drug supplementation were excluded. The detailed data of all subjects are shown in Table I. Clinical experiments were performed with written informed consent from all patients, and the present study was approved by the Ethics Committee of the First People's Hospital (Xianyang, China).

Bone histomorphometry. The proximal tibiae and lumbar vertebrae were decalcified in $0.5 \mathrm{M}$ EDTA $(\mathrm{pH}=8.0)$ and then embedded in paraffin by standard histological procedures. Sections of $5 \mu \mathrm{m}$ in size were cut and stained with hematoxylin and eosin (H\&E), and visualized under a microscope (Olympus Corporation, Tokyo, Japan). In addition, two or three visual fields were selected for bone histomorphometry, and all the images were obtained at a magnification of $x 40$ using a digital camera (Olympus Corporation) that was linked to a desktop computer. A commercial image analysis software, Image Pro Plus 5.1 (Media Cybernetics Inc., Rockville, MD, USA) was used to measure three semi-automatically measured values, namely, bone area, trabecular tissue area and trabecular perimeter. Next, the bone volume fraction (BV/TV), trabecular thickness (Tb.Th), trabecular number (Tb.N) and trabecular space (Tb.Sp) were calculated with the following formulas according to Parfitt's theory (20,21): BV/TV $=100 \times$ B.Ar/T.Ar; $\mathrm{Tb} \cdot \mathrm{Th}=(\mathrm{B} \cdot \mathrm{Ar} / \mathrm{B} \cdot \mathrm{Pm})(\pi / 2) ; \mathrm{Tb} \cdot \mathrm{N}=(\mathrm{B} \cdot \mathrm{Pm} / \mathrm{T} \cdot \mathrm{Ar}) \times 10$; Tb.Sp $=(1,000 \times$ T.Ar-B.Ar $) /$ B.Pm.

Radiographic assessment and symptomatic severity in OA.OA severity was determined using weight-bearing anteroposterior radiographs of the affected knee. Radiographic severity was evaluated according to the Kellgren and Lawrence (KL) grading system (22). In addition, the symptomatic severity of the disease was evaluated according to the Western Ontario McMaster University Osteoarthritis Index (WOMAC), which consists of 3 subscales: Pain, stiffness and physical function (23).

Statistical analysis. The data from these experiments are reported as the mean \pm standard deviation for each group. All statistical analyses were performed using PRISM version 5.0 (GraphPad Software, Inc., La Jolla, CA, USA). The Kolmogorov-Smirnov test was performed to analyse data normality, while an unpaired t-test was used to compare each structural parameter (BV/TV, Tb.N, Tb.Th and Tb.Sp) between the knees of OA patients and healthy controls. The correlation between BV/TV and disease severity classified according to the KL grading system and the correlation between BV/TV and WOMAC scores were determined using Spearman's correlation coefficient. $\mathrm{P}<0.05$ was considered to indicate a statistically significant difference.

\section{Results}

Histological analysis. Histological analysis on trabecular bone in the proximal tibial head and lumbar vertebrae were performed by H\&E staining (Figs. 1 and 2). The histomorphometric section of trabecular bone below the growth plate was markedly different between the healthy control and OA groups. However, the loss of trabecular bone underneath the growth plate in the healthy control group was significantly worse than that of the OA group (Fig. 1). Importantly, H\&E staining demonstrated the increased disconnections and separation of the trabecular bone network as well as the reduction of trabecular bone mass of primary and secondary spongiosa throughout the proximal metaphysis of tibia in the healthy control as compared to the OA group (Fig. 1). It is of note that similar results were observed when the same experiment was repeated on the lumbar vertebrae of healthy control subjects and OA patients from postmenopausal women (Fig. 2).

Bone histomorphometry. The biological properties of trabecular bone were determined at the proximal tibia and lumbar vertebrae. As shown in Table II, patients with OA had higher BV/ TV, Tb.Th, Tb.N values at the proximal tibia compared to that of subjects in the healthy control group (BV/TV, $32.68 \pm 3.54 \%$ vs. $24.75 \pm 2.47 \%, \mathrm{P}=0.002$; Tb.N, $2.54 \pm 0.78 / \mathrm{mm}$ vs. $1.28 \pm 0.46 /$ $\mathrm{mm}, \mathrm{P}=0.001$; Tb.Th, $124.83 \pm 14.83 \mu \mathrm{m}$ vs. $97.23 \pm 8.25 \mu \mathrm{m}$, $\mathrm{P}=0.001)$. Meanwhile, $\mathrm{Tb} . \mathrm{Sp}$ was significantly lower in the $\mathrm{OA}$ group than that of subjects in the healthy control group ( $\mathrm{Tb}$. Sp, $253.68 \pm 22.27 \mu \mathrm{m}$ vs. $347.39 \pm 30.65 \mu \mathrm{m}, \mathrm{P}=0.014)$. As shown in Table III, patients with OA had higher BV/TV, Tb.Th, Tb.N values at the lumbar vertebrae than that of subjects in the healthy control group (BV/TV, $26.37 \pm 2.69 \%$ vs. $20.71 \pm 2.05 \%, \mathrm{P}=0.005$; Tb.N, $2.25 \pm 0.64 / \mathrm{mm}$ vs. $1.62 \pm 0.51 / \mathrm{mm}, \mathrm{P}=0.025$; Tb.Th, $121.49 \pm 12.52 \mu \mathrm{m}$ vs. $80.31 \pm 9.26 \mu \mathrm{m}, \mathrm{P}=0.001)$. Meanwhile, Tb.Sp was significantly lower in the OA group than that of subjects in the healthy control group (Tb.Sp, $312.43 \pm 30.56 \mu \mathrm{m}$ vs. $562.74 \pm 43.67 \mu \mathrm{m}, \mathrm{P}<0.001)$.

Scoring. OA was divided into 3 subgroups according to the $\mathrm{KL}$ grading system. The association of BV/TV at the proximal tibia with radiographic severity and WOMAC scores was illustrated. As shown in Fig. 3A and B, Spearman's rank correlation analysis demonstrated that BV/TV was both positively correlated with radiographic severity and WOMAC scores in the proximal tibia of OA patients $(\mathrm{r}=0.737, \mathrm{P}<0.001$ and $r=0.478, P=0.033$, respectively). Similar results were observed when the same experiment was repeated on the lumbar vertebrae. Spearman's rank correlation analysis demonstrated that $\mathrm{BV} / \mathrm{TV}$ at the lumbar vertebrae was both positively correlated with radiographic severity and WOMAC scores in OA patients $(r=0.842, \mathrm{P}<0.001$ and $\mathrm{r}=0.765, \mathrm{P}=0.033$, respectively, Fig. 3C and D).

\section{Discussion}

The aim of the present study was to determine whether there were altered structural properties of trabecular bone in 
Table I. Detailed data of donors with osteoarthritis or healthy control from postmenopausal women.

\begin{tabular}{|c|c|c|c|c|c|c|}
\hline Subject & Group & $\begin{array}{c}\text { Age } \\
\text { (years) }\end{array}$ & $\begin{array}{l}\text { Height } \\
(\mathrm{cm})\end{array}$ & $\begin{array}{l}\text { BW } \\
(\mathrm{kg})\end{array}$ & $\begin{array}{c}\mathrm{BMD} \\
\left(\mathrm{g} / \mathrm{cm}^{2}\right)\end{array}$ & T-score \\
\hline 1 & OA & 54 & 165 & 65 & 1.09 & -0.39 \\
\hline 2 & OA & 50 & 163 & 72 & 0.96 & -0.57 \\
\hline 3 & $\mathrm{OA}$ & 58 & 158 & 68 & 0.87 & -0.97 \\
\hline 4 & OA & 49 & 155 & 60 & 0.93 & -1.22 \\
\hline 5 & OA & 56 & 168 & 57 & 0.78 & -1.55 \\
\hline 6 & OA & 57 & 156 & 62 & 0.92 & -0.84 \\
\hline 7 & OA & 53 & 164 & 69 & 1.04 & -0.45 \\
\hline 8 & OA & 60 & 158 & 73 & 1.13 & -0.34 \\
\hline 9 & OA & 55 & 167 & 60 & 0.91 & -0.95 \\
\hline 10 & OA & 54 & 163 & 76 & 1.25 & -0.27 \\
\hline 11 & $\mathrm{HC}$ & 52 & 165 & 63 & 0.86 & -1.45 \\
\hline 12 & $\mathrm{HC}$ & 56 & 160 & 70 & 0.79 & -1.78 \\
\hline 13 & $\mathrm{HC}$ & 55 & 162 & 65 & 0.91 & -0.86 \\
\hline 14 & $\mathrm{HC}$ & 50 & 158 & 72 & 0.88 & -1.43 \\
\hline 15 & $\mathrm{HC}$ & 59 & 163 & 62 & 0.79 & -1.74 \\
\hline 16 & $\mathrm{HC}$ & 55 & 164 & 68 & 0.72 & -2.45 \\
\hline 17 & $\mathrm{HC}$ & 56 & 157 & 58 & 0.65 & -2.82 \\
\hline 18 & $\mathrm{HC}$ & 54 & 162 & 63 & 0.81 & -1.69 \\
\hline 19 & $\mathrm{HC}$ & 53 & 160 & 70 & 0.74 & -2.07 \\
\hline 20 & $\mathrm{HC}$ & 54 & 166 & 65 & 0.82 & -1.79 \\
\hline
\end{tabular}

OA, osteoarthritis; $\mathrm{HC}$, healthy control; BW, body weight; $\mathrm{BMD}$, bone mineral density.

proximal tibia and lumbar vertebrae when comparing healthy control subjects and patients with OA in postmenopausal female groups. Compared with the healthy control group, patients with OA had higher BV/TV, Tb.Th and Tb.N values and lower Tb.Sp values in the proximal tibia and lumbar vertebrae. These results demonstrated that a different bone mineralization status in the proximal tibia and lumbar vertebrae existed between healthy control subjects and OA patients from postmenopausal women, and that OA may be associated with an increased BMD.

Previous large epidemiological surveys confirm that radiographic knee OA is associated with high femoral neck BMD $(14,24)$. However, to the best of our knowledge, there are no studies that have simultaneously investigated the association of $\mathrm{OA}$ with the BMD at the proximal tibia and lumbar vertebrae. The present study identified that the correlation of BV/TV with radiographic severity and WOMAC scores at lumbar vertebrae was more closely at proximal tibia. In addition, the study demonstrated marked differences in the histomorphometric sections of some microarchitectural parameters at the proximal tibia and lumbar vertebrae between OA patients and healthy control subjects in postmenopausal women. Moreover, previous studies have demonstrated clear trends of trabecular microarchitectural deterioration during aging in women $(11,25)$. In postmenopausal women, lumbar OA leads to an increase in lumbar spine areal BMD (aBMD), and lumbar spine aBMD increases with the KL grade (26).
Table II. Comparison of bone parameters of proximal tibia between osteoarthritis and healthy controls from postmenopausal women.

\begin{tabular}{lccr}
\hline Bone parameters & OA $(\mathrm{n}=10)$ & $\mathrm{HC}(\mathrm{n}=10)$ & P-value \\
\hline BV/TV $(\%)$ & $32.68 \pm 3.54$ & $24.75 \pm 2.47$ & $0.002^{\mathrm{a}}$ \\
Tb.N $/ \mathrm{mm}$ & $2.54 \pm 0.78$ & $1.28 \pm 0.46$ & $0.001^{\mathrm{a}}$ \\
Tb.Th $(\mu \mathrm{m})$ & $124.83 \pm 14.83$ & $97.23 \pm 8.25$ & $0.001^{\mathrm{a}}$ \\
Tb.Sp $(\mu \mathrm{m})$ & $253.68 \pm 22.27$ & $347.39 \pm 30.65$ & $0.014^{\mathrm{b}}$ \\
\hline
\end{tabular}

OA, osteoarthritis; HC, healthy control; BV/TV, bone volume over total volume; $\mathrm{Tb}$. N, trabecular number; Tb. Th, trabecular thickness; Tb. Sp, trabecular separation. ${ }^{\mathrm{a}} \mathrm{P}<0.001$ and ${ }^{\mathrm{b}} \mathrm{P}<0.05$.

Table III. Comparison of bone parameters of lumbar vertebrae between osteoarthritis and healthy control from postmenopausal women.

\begin{tabular}{lccr}
\hline Bone parameters & OA $(\mathrm{n}=10)$ & $\mathrm{HC}(\mathrm{n}=10)$ & P-value \\
\hline BV/TV $(\%)$ & $26.37 \pm 2.69$ & $20.71 \pm 2.05$ & $0.005^{\mathrm{a}}$ \\
Tb.N $/ \mathrm{mm}$ & $2.25 \pm 0.64$ & $1.62 \pm 0.51$ & $0.025^{\mathrm{b}}$ \\
Tb.Th $(\mu \mathrm{m})$ & $121.49 \pm 12.52$ & $80.31 \pm 9.26$ & $0.001^{\mathrm{a}}$ \\
Tb.Sp $(\mu \mathrm{m})$ & $312.43 \pm 30.56$ & $562.74 \pm 43.67$ & $<0.001^{\text {a }}$ \\
\hline
\end{tabular}

OA, osteoarthritis; HC, healthy control; BV/TV, bone volume over total volume; Tb. N, trabecular number; Tb. Th, trabecular thickness; Tb. Sp, trabecular separation. ${ }^{\mathrm{a}} \mathrm{P}<0.001$ and ${ }^{\mathrm{b}} \mathrm{P}<0.05$.

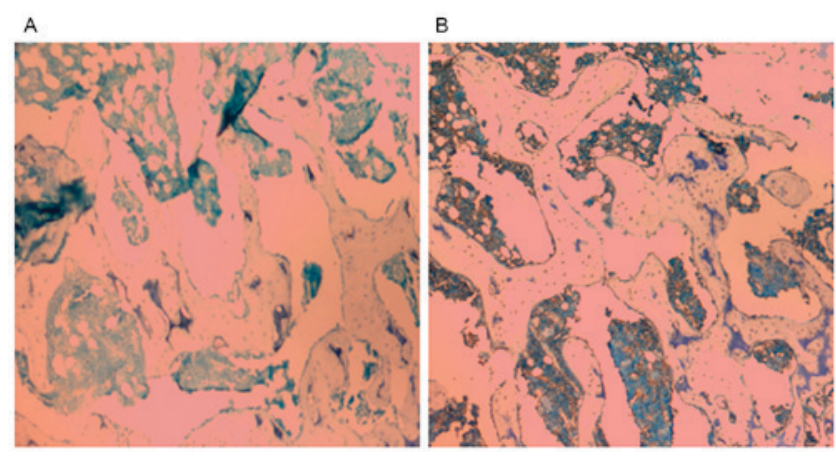

Figure 1. Histomorphometric section of the proximal tibia of (A) a healthy control and (B) osteoarthritis from postmenopausal women.

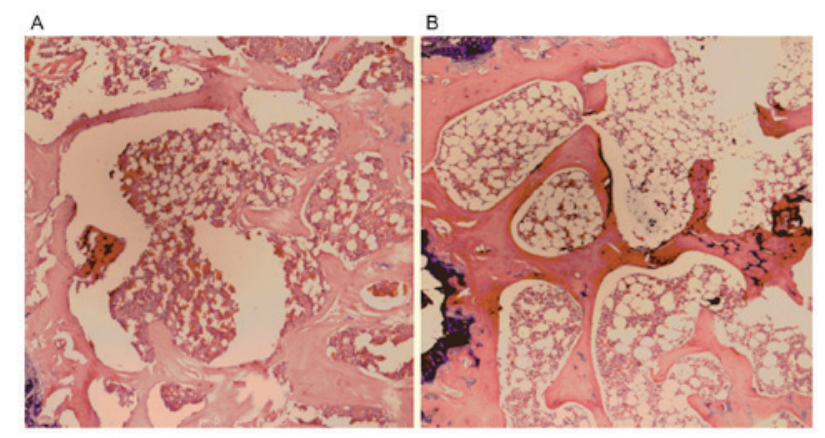

Figure 2. Histomorphometric section of the lumbar vertebrae of (A) a healthy control and (B) osteoarthritis from postmenopausal women. 
A

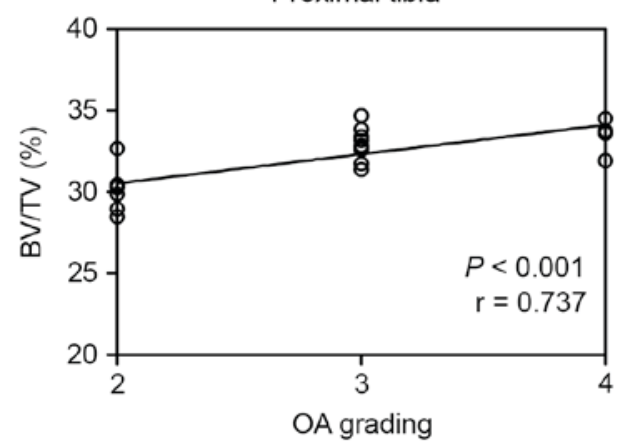

C

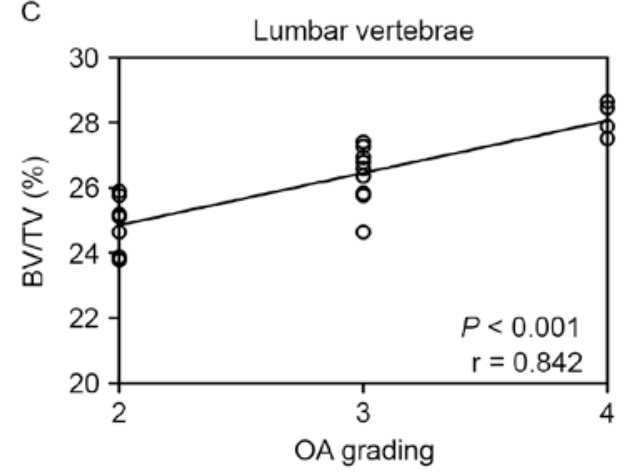

B

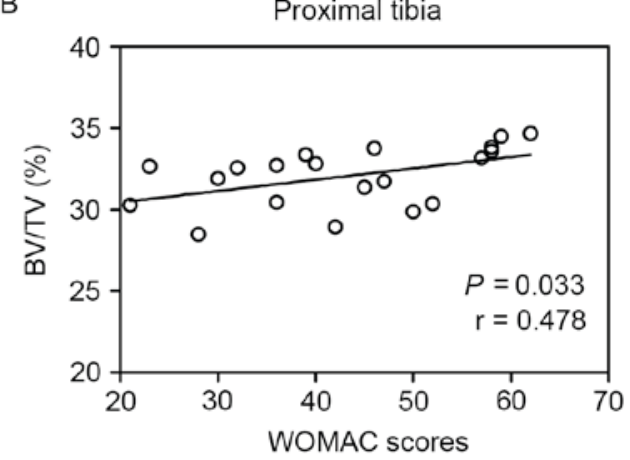

D

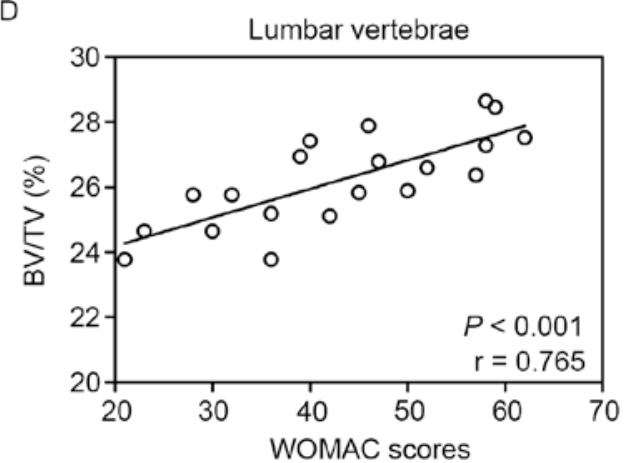

Figure 3. (A) Correlation between BV/TV of proximal tibia in OA patients from postmenopausal women and disease severity classified according to the KL grading system $(\mathrm{r}=0.737, \mathrm{P}<0.001)$. (B) Correlation between BV/TV of proximal tibia in OA patients from postmenopausal women and WOMAC scores $(\mathrm{r}=0.478, \mathrm{P}=0.033)$. (C) Correlation between $\mathrm{BV} / \mathrm{TV}$ of lumbar vertebrae in $\mathrm{OA}$ patients from postmenopausal women and disease severity classified according to the KL grading system ( $\mathrm{r}=0.842, \mathrm{P}<0.001)$. (D) Correlation between $\mathrm{BV} / \mathrm{TV}$ of proximal tibia in OA patients from postmenopausal women and WOMAC scores $(\mathrm{r}=0.765, \mathrm{P}<0.001)$. BV/TV, bone volume over total volume; KL, Kellgren and Lawrence; WOMAC, Western Ontario McMaster University Osteoarthritis Index.

Consistent with previous reports, the present study demonstrated an increased BMD and BV/TV in OA patients. This is in accordance with what was previously identified as knee $\mathrm{OA}$ and was associated with increased hip and even spine aBMD $(27,28)$. Lumbar vertebrae and proximal tibia BV/TV also increase with the KL grade and WOMAC scores in postmenopausal women with OA.

A previous study demonstrated that there was a high prevalence of OA presenting with osteophytes (90\% of patients) and disc space narrowing (65\% of patients) in postmenopausal women aged 74 years on average (18). When OA is defined by the presence of osteophytes, the risk of vertebral fracture has already been shown to be decreased $(29,30)$. Although the risk of vertebral fracture was not performed, the data of the present study demonstrated that there was a significant difference in aBMD between patients with OA and the healthy control. In concordance with the results of the majority of studies concerning BMD measurement, an increased BMD in OA patients was observed. Moreover, an apparent density divided by the total volume was higher in OA because of increased Tb.Th and BV/TV.

Previous comparative research that investigated the association between OA and OP merely emphasized the importance of fracture risk or mechanical properties (31). To the best of our knowledge, this is the first time to evaluate trabecular bone deterioration of the proximal tibia and lumbar vertebrae in postmenopausal women with OA. In addition, the present study revealed differential trabecular structure in proximal tibia and lumbar vertebrae between postmenopausal women with OA and OP, which may be a potential influence on the mechanism of OA and OP. Evidence from the present study supported that there was an important protective mechanism of OA on trabecular microstructure in proximal tibia and lumbar vertebrae from postmenopausal women.

\section{References}

1. Im GI and Kim MK: The relationship between osteoarthritis and osteoporosis. J Bone Miner Metab 32: 101-109, 2014.

2. Wang DM, Zeng QY, Chen SB, Gong Y, Hou ZD and Xiao ZY: Prevalence and risk factors of osteoporosis in patients with ankylosing spondylitis: A 5-year follow-up study of 504 cases. Clin Exp Rheumatol 33: 465-470, 2015.

3. Rossini M, Bagnato G, Frediani B, Iagnocco A, LA Montagna G, Minisola G, Caminiti M, Varenna M and Adami S: Relationship of focal erosions, bone mineral density, and parathyroid hormone in rheumatoid arthritis. J Rheumatol 38: 997-1002, 2011.

4. Steinbeck MJ, Eisenhauer PT, Maltenfort MG, Parvizi J and Freeman TA: Identifying patient-specific pathology in osteoarthritis development based on microCT analysis of subchondral trabecular bone. J Arthroplasty 31: 269-277, 2016.

5. Hada S, Kaneko H, Sadatsuki R, Liu L, Futami I, Kinoshita M, Yusup A, Saita Y, Takazawa Y, Ikeda H, et al: The degeneration and destruction of femoral articular cartilage shows a greater degree of deterioration than that of the tibial and patellar articular cartilage in early stage knee osteoarthritis: A cross-sectional study. Osteoarthritis Cartilage 22: 1583-1589, 2014.

6. Yamamoto Y, Turkiewicz A, Wingstrand $\mathrm{H}$ and Englund M: Fragility fractures in patients with rheumatoid arthritis and osteoarthritis compared with the general population. J Rheumatol 42: 2055-2058, 2015. 
7. Chan MY, Center JR, Eisman JA and Nguyen TV: Bone mineral density and association of osteoarthritis with fracture risk. Osteoarthritis Cartilage 22: 1251-1258, 2014.

8. Ramonda R, Sartori L, Ortolan A, Frallonardo P, Lorenzin M, Punzi L and Musacchio E: The controversial relationship between osteoarthritis and osteoporosis: An update on hand subtypes. Int J Rheum Dis 19: 954-960, 2016.

9. Lethbridge-Cejku M, Tobin JD, Scott WW Jr, Reichle R, Roy TA, Plato CC and Hochberg MC: Axial and hip bone mineral density and radiographic changes of osteoarthritis of the knee: Data from the baltimore longitudinal study of aging. J Rheumatol 23: 1943-1947, 1996.

10. Rubinacci A, Tresoldi D, Scalco E, Villa I, Adorni F, Moro GL Fraschini GF and Rizzo G: Comparative high-resolution pQCT analysis of femoral neck indicates different bone mass distribution in osteoporosis and osteoarthritis. Osteoporos Int 23: 1967-1975, 2012.

11. Djuric M,Zagorac S, Milovanovic P, Djonic D, Nikolic S, Hahn M, Zivkovic V, Bumbasirevic M, Amling $M$ and Marshall RP: Enhanced trabecular micro-architecture of the femoral neck in hip osteoarthritis vs. healthy controls: A micro-computer tomography study in postmenopausal women. Int Orthop 37: 21-26, 2013.

12. Papaloucas CD, Ward RJ, Tonkin CJ and Buckland-Wright C: Cancellous bone changes in hip osteoarthritis: A short-term longitudinal study using fractal signature analysis. Osteoarthritis Cartilage 13: 998-1003, 2005.

13. Makinen TJ, Alm JJ, Laine H, Svedström E and Aro HT: The incidence of osteopenia and osteoporosis in women with hip osteoarthritis scheduled for cementless total joint replacement. Bone 40: 1041-1047, 2007.

14. Multanen J, Heinonen A, Häkkinen A, Kautiainen H, Kujala UM, Lammentausta E, Jämsä T, Kiviranta I and Nieminen MT: Bone and cartilage characteristics in postmenopausal women with mild knee radiographic osteoarthritis and those without radiographic osteoarthritis. J Musculoskelet Neuronal Interact 15: 69-77, 2015

15. Bijlsma JW, Berenbaum F and Lafeber FP: Osteoarthritis: An update with relevance for clinical practice. Lancet 377 2115-2126, 2011

16. Shen Y, Zhang ZM, Jiang SD, Jiang LS and Dai LY Postmenopausal women with osteoarthritis and osteoporosis show different ultrastructural characteristics of trabecular bone of the femoral head. BMC Musculoskelet Disord 10: 35, 2009.

17. Zhang ZM, Jiang LS, Jiang SD and Dai LY: Differential articular calcified cartilage and subchondral bone in postmenopausal women with osteoarthritis and osteoporosis: Two-dimensional analysis. Joint Bone Spine 76: 674-679, 2009.

18. Roux C, Fechtenbaum J, Briot K, Cropet C, Liu-Leage S and Marcelli C: Inverse relationship between vertebral fractures and spine osteoarthritis in postmenopausal women with osteoporosis. Ann Rheum Dis 67: 224-228, 2008.

19. Wu CW, Morrell MR, Heinze E, Concoff AL, Wollaston SJ Arnold EL, Singh R, Charles C, Skovrun ML, FitzGerald JD, et al Validation of American College of Rheumatology classification criteria for knee osteoarthritis using arthroscopically defined cartilage damage scores. Semin Arthritis Rheum 35: 197-201, 2005.
20. Parfitt AM, Drezner MK, Glorieux FH, Kanis JA, Malluche H, Meunier PJ, Ott SM and Recker RR: Bone histomorphometry: Standardization of nomenclature, symbols, and units. Report of the ASBMR Histomorphometry Nomenclature Committee. J Bone Miner Res 2: 595-610, 1987.

21. Dempster DW, Compston JE, Drezner MK, Glorieux FH, Kanis JA, Malluche H, Meunier PJ, Ott SM, Recker RR and Parfitt AM: Standardized nomenclature, symbols, and units for bone histomorphometry: A 2012 update of the report of the ASBMR histomorphometry nomenclature committee. J Bone Miner Res 28: 2-17, 2013.

22. Kellgren JH and Lawrence JS: Radiological assessment of osteo-arthrosis. Ann Rheum Dis 16: 494-502, 1957.

23. Bellamy N, Buchanan WW, Goldsmith $\mathrm{CH}$, Campbell J and Stitt LW: Validation study of WOMAC: A health status instrument for measuring clinically important patient relevant outcomes to antirheumatic drug therapy in patients with osteoarthritis of the hip or knee. J Rheumatol 15: 1833-1840, 1988.

24. Sowers M, Lachance L, Jamadar D, Hochberg MC, Hollis B, Crutchfield M and Jannausch ML: The associations of bone mineral density and bone turnover markers with osteoarthritis of the hand and knee in pre- and perimenopausal women. Arthritis Rheum 42: 483-489, 1999.

25. Milovanovic P, Djonic D, Marshall RP, Hahn M, Nikolic S, Zivkovic V, Amling M and Djuric M: Micro-structural basis for particular vulnerability of the superolateral neck trabecular bone in the postmenopausal women with hip fractures. Bone 50: 63-68, 2012.

26. Kolta S, Briot K, Fechtenbaum J, Paternotte S, Armbrecht G, Felsenberg D, Glüer CC, Eastell R and Roux C: TBS result is not affected by lumbar spine osteoarthritis. Osteoporos Int 25: 1759-1764, 2014.

27. Hart DJ, Mootoosamy I, Doyle DV and Spector TD: The relationship between osteoarthritis and osteoporosis in the general population: The Chingford Study. Ann Rheum Dis 53: 158-162, 1994.

28. Jiang LS, Zhang ZM, Jiang SD, Chen WH and Dai LY: Differential bone metabolism between postmenopausal women with osteoarthritis and osteoporosis. J Bone Miner Res 23: 475-483, 2008

29. Arden NK, Griffiths GO, Hart DJ, Doyle DV and Spector TD: The association between osteoarthritis and osteoporotic fracture: The Chingford Study. Br J Rheumatol 35: 1299-1304, 1996.

30. Jones G, White C, Nguyen T, Sambrook PN, Kelly PJ and Eisman JA: Prevalent vertebral deformities: Relationship to bone mineral density and spinal osteophytosis in elderly men and women. Osteoporos Int 6: 233-239, 1996.

31. Li B and Aspden RM: Composition and mechanical properties of cancellous bone from the femoral head of patients with osteoporosis or osteoarthritis. J Bone Miner Res 12: 641-651, 1997. 